\title{
Design of circular flexible plates under of corrosion wear
}

\author{
Vladimir Mavzovin ${ }^{1 *}$ and Igor Ovchinnikov ${ }^{2}$ \\ ${ }^{1}$ Moscow State University of Civil Engineering, Yaroslavskoe shosse, 26, Moscow, 129337, Russia \\ ${ }^{2}$ Tyumen Industrial University, Perm National Research Polytechnic University, Russia
}

\begin{abstract}
The problems of optimal design of metal structures are usually formulated as the problem of finding such values of the selected parameters of structures that provide the smallest (or largest) value of the selected optimality criterion in the area of admissible design solutions. In most works on the optimization of metal structures, parametric problems are considered, limited by the preliminary assignment of a constructive form with the possibility of changing its parameters. To solve these problems, both deterministic and probabilistic optimization models can be used. A deterministic problem of optimal design of flexible round metal plates exposed to the combined action of a load and an aggressive environment causing corrosive wear of one of the plate surfaces is considered. A feature of the statement is that it takes into account the effect of the stressed state of the plates on the kinetics of their corrosive wear. Due to the insufficient efficiency of random search methods, the sequential quadratic programming technique is used. Two formulations of the optimal design problem are formulated, which are reduced to the minimax problem. 8 optimization projects were considered, their comparative analysis was carried out. It is shown that the realization of the found optimal thickness profiles leads either to a significant decrease in the stress level at the end of the plate's service life, or to a significant increase in the service life. Calculation results show that maximum fatigue life designs are equal at end-of-life, and the maximum increase in fatigue life for hardpinned inserts along the contour is $59 \%$. As a result of the study, it was found that the problems of optimal design of flexible round plates under conditions of corrosive wear (the rate of which depends on the level of stresses in the plates), presented in the form of a minimax problem, can be effectively solved using the sequential quadratic programming technique, and the study of time evolution is intense -strain state can be carried out using a combination of the mesh method and the Adams method. Realization of the found optimal thickness profiles leads either to a significant decrease in the stress level at the end of the plate's service life, or to a significant increase in the service life.
\end{abstract}

*Corresponding author: mavzvin@mail.ru 


\section{Introduction}

Corrosion wear is usually modeled by the decrease in the initial thickness of thin-walled metal structural elements over time, caused by the chemical interaction of the surface of the elements with an aggressive operating environment. Under the influence of corrosion, according to [1], about $27.5 \%$ of metal structures fail, which exceeds all other types of impacts. Corrosion wear is usually described by the kinetics of changing the depth of the corrosion layer (or by the kinetics of decreasing the thickness of corrosive elements). Since this characteristic can be easily observed and measured, the experimental data on the measurement of the kinetics of corrosive wear are sufficiently reliable for their use in various problems. In addition, the effect of corrosive wear on the stress state is significant for thin-walled structures. These reasons were the main ones when choosing a target and object of a thin-walled structure for optimal design - a thin round plate subject to corrosive wear, leading to a change in its stress-strain state and an increase in its flexibility with the need to take into account geometric nonlinearity.

The problems of optimal design of metal structures are usually formulated as the problem of finding such values of the selected parameters of structures that provide the smallest (or largest) value of the selected optimality criterion in the area of admissible design solutions. In most works on the optimization of metal structures, parametric problems are considered, limited by the preliminary assignment of a constructive form with the possibility of changing its parameters. To solve these problems, both deterministic and probabilistic optimization models can be used. Recently, in order to bring the formulations of optimization problems closer to reality, they began to take into account the destructive effect of the operating environment. A sufficiently detailed analysis of the state of the problem of the optimal design of metal structures taking into account the operating conditions (in particular, corrosive wear) is given in the work of the authors. [2], so here we do not consider this issue in detail.

\section{Methods}

In most works on the optimization of loaded structures under aggressive conditions [1], the design parameters are several numerical parameters, in particular, the shell radius, rib thickness, number of ribs, etc. We consider the problem of designing a function of the thickness of the plate from the radius. Known numerical and analytical methods for solving such problems in the case of an inert environment [3,4]. The need to take into account corrosive wear limits the applicability of analytical approaches and leads to a sharp increase in the computational complexity of the optimization problem when solving it using nonlinear programming methods. The random search methods [1] are not efficient enough in the problems of parametric minimization of high dimension, generated by the original problem of minimizing the functional. Therefore, this work uses the sequential quadratic programming technique, that is, the SQP-method [5], which is a fairly effective method for analyzing extremal problems of sufficiently large dimension for smooth objective functions [6].

Further, round flexible plates with an axisymmetric distribution of variable thickness are considered. One of the 4 standard boundary conditions acts on the edge [6], it is assumed that a uniformly distributed load is applied to the upper surface, and an aggressive medium acts on the lower surface (Fig. 1). 


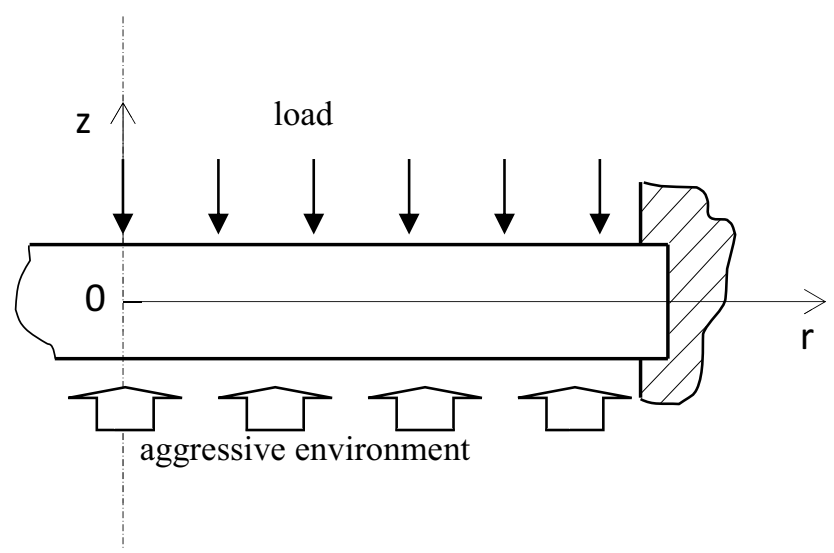

Fig. 1. Right half of the cross section form:

The plate bending equations in the framework of the Kirchhoff theory have the

$$
\begin{aligned}
& u^{\prime \prime}+\frac{1}{r} u-\frac{1}{r^{2}} u=-\frac{D_{n}^{\prime}}{D_{n}}\left(u^{\prime}+\frac{1}{2} \varphi^{2}+\frac{v}{r} u\right)-\frac{1-v}{2 r} \varphi^{2}-\varphi \varphi^{\prime} \\
& \varphi^{\prime \prime}+\frac{1}{r} \varphi^{\prime}-\frac{1}{r^{2}} \varphi=-\frac{D_{m}^{\prime}}{D_{m}}\left(\varphi^{\prime}+\frac{v}{r} \varphi\right)+\varphi \frac{D_{n}}{D_{m}}\left(u^{\prime}+\frac{1}{2} \varphi^{2}+\frac{v}{r} u\right)-\frac{1}{D_{m} r} \int_{0}^{r} q r d r
\end{aligned}
$$

Here: is the radius of the considered point of the middle surface of the plate, is the radial and vertical displacement, respectively, is the modulus of elasticity of the plate material, is the coefficient of transverse deformation, is the thickness of the plate variable along the radius, is the intensity of the transverse load acting on the plate,

$D_{m}=E h^{3} / 12\left(1-v^{2}\right), D_{n}=E h /\left(1-v^{2}\right)$, - lexural and tangential stiffness. Standard boundary conditions include:

$$
\begin{gathered}
\varphi(R)=0, u(R)=0-\text { hard substitution } \\
\varphi(R)=0, N_{r}(R)=0-\text { sliding termination } \\
M_{r}(R)=0, u(R)=0-\text { articulated support } \\
M_{r}(R)=0, N_{r}(R)=0-\text { free support }(2) .
\end{gathered}
$$

Where $\mathrm{M}_{\mathrm{r}}, \mathrm{N}_{\mathrm{r}}$ - are the moments and forces in the circular section of the plate. The latter, together with the moments and forces in the radial section $M_{t}, N_{t}$, are found by solving system (1), (2) using the expressions:

$$
M_{r}=D_{m}\left(\varphi^{\prime}+\frac{v}{r} \varphi\right)
$$




$$
\begin{array}{r}
N_{r}=D_{n}\left(u^{\prime}+\frac{1}{2} \varphi^{2}+\frac{v}{r} u\right) \\
M_{t}=D_{m}\left(v \varphi^{\prime}+\frac{1}{r} \varphi\right), \\
N_{r}=D_{n}\left(v u^{\prime}+\frac{v}{2} \varphi^{2}+\frac{1}{r} u\right)
\end{array}
$$

The criterion of local strength is introduced $\sigma_{u}(r)<[\sigma]$, where $\sigma_{u}(r)=$ $\max \left[\sigma_{u}^{-}(r), \sigma_{u}^{+}(r), \sigma_{u}^{0}(r)\right]$, and the subscripts '-', '+', '0' correspond to the intensity of stresses on the lower, upper and middle surfaces of the plate:

$$
\sigma_{u}^{*}=\frac{1}{\sqrt{2}} \sqrt{\left(\sigma_{r}^{*}-\sigma_{t}^{*}\right)^{2}+\sigma_{t}^{* 2}+6 \tau_{r z}^{* 2}}
$$

where the index is entered $\quad * "=\left\{{ }^{\prime}-,^{\prime}+{ }^{\prime}, 0^{\prime}\right\}$, and stresses are calculated by the formulas:

$$
\begin{gathered}
\sigma_{r}^{ \pm}=\frac{N_{r}}{h} \pm \frac{6 M_{r}}{h^{2}}, \\
\sigma_{t}^{ \pm}=\frac{N_{t}}{h} \pm \frac{6 M_{t}}{h^{2}}, \\
\tau_{r z}^{ \pm}=0, \\
\sigma_{r}^{0}=\frac{N_{r}}{h}, \\
\sigma_{t}^{0}=\frac{N_{t}}{h}, \\
\tau_{r z}^{0}=\frac{3 q r}{4 h} \pi r^{2}
\end{gathered}
$$

The shear stress in the circumferential section, which is neglected in the Kirchhoff theory, is taken into account by us in accordance with the recommendation in [6].

The corrosive wear rate is calculated according to the V. Dolinsky [1]:

$$
\frac{d h}{d t}=-A\left(1+k \sigma_{u}^{-}\right)(6)
$$

where $\mathrm{A}$ is the corrosion rate in the unstressed state, $k$ is the coefficient taking into account the accelerating effect of stresses on the corrosion rate.

The above equations form a closed system that describes the process of deformation of the plate in time, taking into account the kinetics of corrosion wear.

The system was solved in accordance with the following algorithm. For each moment of time under consideration, with the law of change known from the previous step, the linearization of equations (1) according to Newton was applied. The solution of a series of linearized equations was carried out by the finite-difference method, for which a frequent grid was set at the radius $\{r\}_{1}$. The time evolution of the thickness system on a dense grid $\{h(t)\}_{1}$ was found by the Adams method. The initial values of $\{h(0)\}_{1}$ were determined by interpolation over the initial thicknesses $\{H\}_{2}$, given on some sparse mesh $\{r\}_{2}$. Finally, system $\{H\}_{2}$ was proposed as variable parameters for the SQP-method. 
Based on the features of the SQP-method [4], the extremal problem should be reformulated in such a way as to transfer the complexity of the relief from the constraints to the objective function. Therefore, we used two problem statements:

- First performance. Among all flexible plates having the same initial volume $V=V_{c}$ find one in which the maximum stress intensity $\max _{r}\left\{\sigma_{u}\right\}_{1}$ becomes minimum at the end of a given time interval $\mathrm{T}_{\text {end. }}$.

- Second production. Among all flexible plates with the same initial volume $\mathrm{V}=\mathrm{V}_{\mathrm{c}}$ find one in which the maximum stress intensity $\max _{r}\left\{\sigma_{u}\right\}_{1}$ becomes the limit $[\sigma]$ later than all.

Introducing a system of times $\{t\}_{1}$, for the second setting, which will determine the times of reaching the limiting values $[\sigma]$ by each of the grid values $\left\{\sigma_{u}\right\}_{1}$, we obtain that both statements lead to the minimax problem:

$$
\text { find } h^{*}=\arg \min \max \{f(h)\}_{1} \text { with restrictin } V(h)-V_{c}=0,(7)
$$

in which $\{f\} \equiv\left\{\sigma_{u}\right\} \quad$ or the first setting and $\{f\} \equiv\{t\}$ for the second. Objective function $\max _{f}\{f\}_{1}$ non smooth, but the specific structure of discontinuities in its gradient made it possible to supplement the standard SQP-method with a simple procedure, the idea of which is given in [4]. This procedure simulates a smooth objective function for the SQPmethod when it solves minimax problems.

\section{Calculation results}

To cover various options for the development of deformation processes, equations (1) - (6) were reduced to a dimensionless form, for which, among others, the following dimensionless parameters were introduced:

$$
\begin{aligned}
& \bar{q}=q \frac{E\left(h^{p}\right)^{4}}{R^{4}}-\text { dimensionless load, } \\
& \alpha=k \max _{r}\left\{\sigma_{u}^{p}\right\}_{1} \text { - parameter reflecting the acceleration of corrosion under stress, } \\
& \beta=\frac{h^{p}}{A[T]}-\text { relative reduction in thickness, } \\
& \gamma=\frac{[\sigma]}{\max _{r}\left\{\sigma_{u}^{p}\right\}_{1}} \text { - parameter characterizing the relative margin of safety. }
\end{aligned}
$$

Here $\mathrm{R}$ - is the radius of the plate, [T] - is the specified durability, and the index " $p$ " marks the initial parameters for a plate of constant thickness with an initial volume $\mathrm{V}_{\mathrm{c}}$. Below in table. 1 shows the quality indicators of some optimal designs of plates with hard pinching: 
Table 1. Quality indicators of some optimal plate designs with hard pinching

\begin{tabular}{|c|c|c|c|c|c|c|c|c|}
\hline & \multicolumn{4}{|c|}{ First production, $\beta=7.4$} & \multicolumn{4}{|c|}{ Second production, $\gamma=2.3$} \\
\hline value $\alpha$ & 0.25 & & 0.71 & & 0.25 & & 0.71 & \\
\hline Project designation & $\mathbf{A}$ & $\mathbf{B}$ & $\mathbf{C}$ & $\mathbf{D}$ & $\mathbf{E}$ & $\mathbf{F}$ & $\mathbf{G}$ & $\mathbf{H}$ \\
\hline Load $\bar{q}_{\text {initial }}$ & 0.43 & 15.6 & 0.43 & 15.6 & 0.43 & 15.6 & 0.43 & 15.6 \\
\hline $\begin{array}{l}\text { End deflection in } \\
\text { the center } \\
w(0)_{\text {end }} /\left\langle h_{\text {end }}\right\rangle\end{array}$ & 0.121 & 2.23 & 0.144 & 2.79 & 0.132 & 2.66 & 0.166 & 2.94 \\
\hline value $K_{\sigma}$ & 0.41 & 0.39 & 0.45 & 0.41 & 1 & 1 & 1 & 1 \\
\hline value $K_{T}$ & 1 & 1 & 1 & 1 & 1.42 & 1.55 & 1.44 & 1.59 \\
\hline
\end{tabular}

For a comparative analysis of the calculation results, the following characteristics were introduced:

- Stress relaxation index $K_{\sigma}$, which is the ratio of the maximum stress intensity in the optimal plate at the end of its service life to the same stress intensity for a plate of constant thickness with the same initial volume and the same service life.

- The index of the increase in the durability of $K_{T}$, which is the ratio of the durability of the optimal flexible plate to the durability of a flexible plate of constant thickness with the same initial volume and the same ultimate strength.

Figure 2 shows the kinetics of change in characteristics for project G. The initial thickness profile of the optimal design can be estimated from Figure $2 \mathrm{a}$.

a)

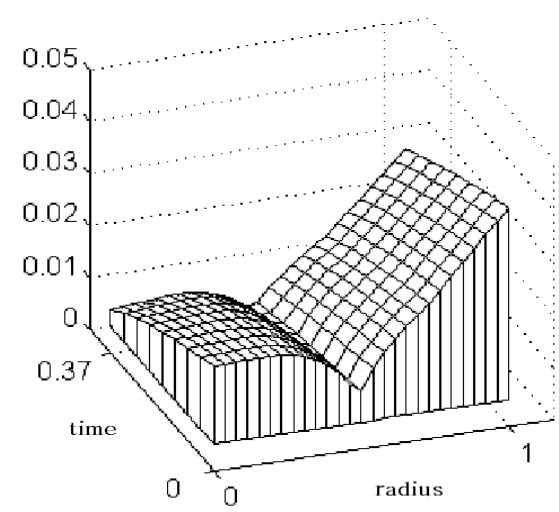

б)

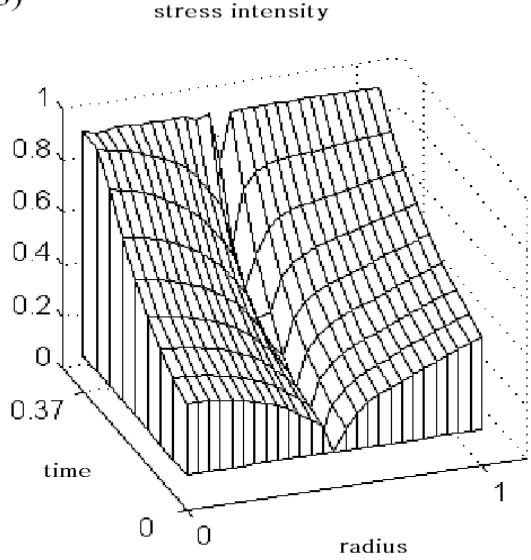

Fig. 2. Kinetics of changes in characteristics for the project

\section{Discussion}

The calculation results show that the designs of maximum durability (setting 2) are equal at the end of their service life (Fig.2b), and the maximum increase in durability for plates with rigid pinching along the contour is $59 \%$ (design $\mathrm{H}$ ). The practical feasibility of these projects is confirmed by research [8]. 
It should be noted that this study used the model of corrosive wear (6) proposed by V.M. Dolinsky. In this model, the effect of the stress state on the corrosion rate is taken into account by introducing a stress intensity function. In other models of corrosive wear, the effect of the stress state can be taken into account by introducing a function of other invariants of the stress-strain state, for example, average stress or specific energy of deformation. In such cases, the results may be obtained slightly different from those presented in this work.

In addition, several other statements of the optimal design problem can be formulated. For example, it can be required that at the end of a given service life the plate has an optimal shape according to some criteria. In this case, first, this optimal shape of the plate is determined, and then the corrosive wear is switched on "vice versa", that is, in the process of numerical modeling of the behavior of the plate in time, material is not removed from the surface of the plate due to corrosion, but on the contrary, the material is "layered" on the surface according to the corrosive wear model (6) and then after a time equal to the specified service life, we will obtain the shape of the plate at the initial moment of time. Obviously, at the initial moment of time, this plate will not have the optimal shape, but it will acquire the optimal shape by the end of the specified service life. Another formulation of the optimal design problem can be the formulation when, by specifying the initial shape of the plate, it is required to achieve the lowest possible material consumption for corrosion during operation in a given aggressive environment.

\section{Conclusions}

1. The problems of optimal design of flexible round plates under conditions of corrosive wear, presented in the form of a minimax problem, admit an effective solution using the sequential quadratic programming technique.

2. The study of the time evolution of the stress-strain state can be carried out using a combination of the mesh method and the Adams method.

3. Realization of the found optimal thickness profiles leads either to a significant decrease in the stress level at the end of the plate's service life, or to a significant increase in the service life.

4. In addition to the one considered, other formulations of optimal design problems are possible, for example, the achievement of the optimal shape of a plate subject to corrosive wear at the end of a given service life.

\section{References}

1. I.G. Ovchinnikov, Yu.M. Pochtman Thin-walled structures under corrosive wear conditions. Calculation and optimization 192 (1995)

2. I.G. Ovchinnikov, V.S. Mavzovin Engineering and construction bulletin of the Caspian region: scientific and technical journal 1 (31), 43 (2020)

3. V.P. Malkov, A.G. Ugodchikov Optimization of elastic systems 228 (198)

4. N.V. Banichuk An introduction to design optimization 301 (1986)

5. F. Gill, W. Murray, M. Wright Practical optimization 509 (1985)

6. W.Hock, K. Schittowski Computing (30), 335 (1983)

7. S.P. Timoshenko, S Voinovsky-Krieger Plates and shells 636 (1963)

8. I.G. Ovchinnikov, A.V. Bochkarev Izvestiya vuzov. Construction. (6), 10 (2003) 